\title{
Erratum to: Conformational Transition Pathway in the Inhibitor Binding Process of Human Monoacylglycerol Lipase
}

\author{
Huayou Chen $\cdot$ Rui Tian $\cdot$ Zhong Ni \\ Zhongge Zhang $\cdot$ Ake Vastermark . \\ Hongzhang Chen $\cdot$ Qi Guo
}

Published online: 7 October 2014

(C) Springer Science+Business Media New York 2014

\section{Erratum to: Protein J}

\section{DOI 10.1007/s10930-014-9572-z}

The authors would like to make the following corrections:

Huayou Chen and Zhong $\mathrm{Ni}$ are now the co-corresponding authors of this original article. Milton H. Saier has been removed as an author from this original article and is also no longer a corresponding author. Ake Vastermark has been added as a co-author to the original article.

The online version of the original article can be found under doi:10. 1007/s10930-014-9572-z.

H. Chen $\cdot$ R. Tian $\cdot$ Z. Ni $(\bowtie) \cdot$ Q. Guo

Institute of Life Sciences, Jiangsu University, Zhenjiang 212013,

China

e-mail: nizhong@usj.edu.cn

H. Chen $\cdot$ Z. Zhang $\cdot$ A. Vastermark

Division of Biological Sciences, University of California at San

Diego, La Jolla, CA 92093-0116, USA

H. Chen $(\bowtie) \cdot$ H. Chen

National Key Laboratory of Biochemical Engineering, Institute

of Process Engineering, Chinese Academy of Sciences,

Beijing 10090, China

e-mail: phdc@163.com 